\title{
MIMO-Filtered OFDM System Improvement Using Modified Concatenated RS/LDPC Codes
}

Ghasan Ali Hussain ( $\square$ ghasan.alabaichy@uokufa.edu.iq )

Faculty of Engineering, University of Kufa https://orcid.org/0000-0001-8368-4708

\section{Research Article}

Keywords: f-OFDM, 5G, MIMO, RS, LDPC, BER, PAPR, OOBE

Posted Date: November 1st, 2021

DOI: https://doi.org/10.21203/rs.3.rs-1022604/v1

License: (9) This work is licensed under a Creative Commons Attribution 4.0 International License. Read Full License 


\title{
MIMO-Filtered OFDM System Improvement Using Modified Concatenated RS/LDPC Codes
}

\author{
Ghasan Ali Hussain*
}

Received: date / Accepted: date

\begin{abstract}
In mobile communication systems, there are errors that will be generated in the digital signal due to fading and interference. Consequently, different techniques are used to improve the system's reliability and enhance the signal's robustness. Channel coding techniques are used to enhance the system reliability of $5 \mathrm{G}$ wireless communication systems. In the upcoming wireless technologies, LDPC codes are still introduced as an alternative to turbo codes. However, the error floor phenomenon is one of the biggest demerits of using LDPC code in the different communication systems that need low error rates. This paper uses RS codes with LDPC codes in a concatenated code to solve this demerit of LDPC codes. Meanwhile, a modified concatenated RS/LDPC codes are created using outer RS codes with inner LDPC codes then appended by interleaver, unlike the conventional concatenated codes that use the interleaver between both codes. Thereafter, the modified concatenated RS/LDPC codes were suggested to enhance BER performance for the f-OFDM system. The results showed that using the proposed concatenated code outperformed using single and familiar concatenated RS/LDPC code in terms of improving BER performance. Meanwhile, the proposed system achieved lower OOBE values than the conventional OFDM system. Therefore, the resulted system can be introduced as a competitor candidate for $5 \mathrm{G}$ wireless communication systems due to these features.
\end{abstract}

Keywords f-OFDM $\cdot 5 \mathrm{G} \cdot \mathrm{MIMO} \cdot \mathrm{RS} \cdot \mathrm{LDPC} \cdot \mathrm{BER} \cdot \mathrm{PAPR} \cdot \mathrm{OOBE}$

\section{Introduction}

In broadband wireless communication systems including the Fourth Generation (4G), the technology of Multicarrier Modulation (MCM) especially Orthogonal Frequency Division Multiplexing (OFDM) system, is considered a key technology.

Ghasan Ali Hussai

- Department of Electrical Engineering, Faculty of Engineering, University of Kufa, IRAQ. Tel.: +9647801296162

E-mail: ghasan.alabaichy@uokufa.edu.iq 
The design of the OFDM system is suitable to resist the impact of multipath reception. It considerably alleviates the Intersymbol Interference (ISI) and enhances spectral efficiency. Nevertheless, Long Term Evolution (LTE)/4G systems became insufficient in meeting the requirement of wireless communication systems and their requirements of higher data rate due to the rapid growth of wireless communication services. Thereby, Fifth Generation (5G) systems have become a hotspot for research nowadays. On the other hand, due to the high Peak to Average Power Ratio (PAPR) and Out Of Band Emission (OOBE), OFDM does not suitable for the demand of the $5 \mathrm{G}$ heterogeneous service. Moreover, in the entire bandwidth, the OFDM supports one kind of waveform parameter. While, filtered OFDM (f-OFDM) system divides the whole system's bandwidth for several subbands which are filtered using different filters, thereby it supports various waveform parameters based on different service scenarios [1]. In mobile communication systems, errors will be generating in the digital signal due to fading and interference. Consequently, different techniques are used to improve the system reliability and enhance the signal robustness such as increase the transmit signal power and using error detection/correction techniques. However, channel coding techniques are considered a common method to minimize the system error rate [2]. Different channel coding techniques including Bose-Chaudhuri-Hocquenghem (BCH), Reed Solomon (RS), polar, Low Density Parity Check (LDPC), turbo and convolutional codes were suggested by [3-12] to enhance the system reliability $5 \mathrm{G}$ wireless communication system. Meanwhile, LDPC and polar codes were introduced by Third Generation Partnership Project (3GPP) in the recent finalized release-15 5G New Radio (NR) access technology standard to use rather than the turbo and convolutional codes with 4G/LTE [13]. Both polar and LDPC codes are discussed in [14] for the 5G NR standard. They showed that the NR LDPC codes' performance is similar to LTE turbo codes. Nevertheless, turbo codes suffer from error floor at high code rates. On other the hand, NR LDPC codes outperform LTE turbo codes in terms of lower latency and higher throughput[14]. Meanwhile, different channel coding such as convolutional, turbo, LDPC and polar codes have been evaluated in [15] to propose the optimum code for a $5 \mathrm{G}$ mobile communication system over Addictive White Gaussian Noise (AWGN) channel and Binary Phase Shift Keying (BPSK) modulation scheme with $1 / 2$ code rate. The analysis has been performed based on the reliability, flexibility, latency and complexity for short-length message transmission. They showed that the systematic convolutional code outperforms in higher reliability, low encoding latency and better flexibility for the $5 \mathrm{G}$ mobile communication system. While, polar code outperformed in terms of decoding complexity [15].

In 5G technologies, the demand for higher data rates (around 1000 times higher than $4 \mathrm{G}$ ) and higher reliability led to the search for channel coding techniques charactering high efficiency. In the upcoming wireless technologies like Ultra-Reliable Low Latency Communication (URLLC), massive Machine Type Communication (mMTC) and Device to Device communication (D2D) etc, LDPC codes are still introduced as an alternative to turbo codes [16]. It is a type of linear block code has capability of error correction close to Shannon limit [17].However, the error rate floor phenomenon is considered one of the biggest demerits of using LDPC code in different communication systems which needs to low error rates [18-20]. In contrast, different researchers are trying to solve this problem using several techniques. Nevertheless, they are facing the problem of increasing the decoding 
latency or encoding/decoding complexity due to the use of these techniques [21]. On the other hand, RS codes are classical linear block codes utilized in many classical transmissions which required to correct burst errors [22]. Many researchers such as [23-26], proposed using RS with LDPC codes in a concatenated code to solve the problem of the error rate floor phenomenon of LDPC codes. They showed that, the error floor LDPC code could be significantly eliminated by using with outer RS codes in concatenated RS/LDPC codes, achieving high decoding performance. Furthermore, the concatenated RS/LDPC codes have good handling with burst error and improve coding gains owing to use outer RS codes. In digital communication systems, concatenated coding is considered a common error correction technique that uses both codes, outer and inner separating them with interleaver. Using the interleaver is important to scatter burst errors that may occur after the inner decoder and thus helps the outer decodes in correcting the remaining errors from the inner decoder [27]. In [28], a novel BCS code was created using the same idea of concatenated codes with different from combining both codes, then appended by interleaver as shown in Fig. 1. They showed that the proposed code is outperformed the familiar concatenated code.

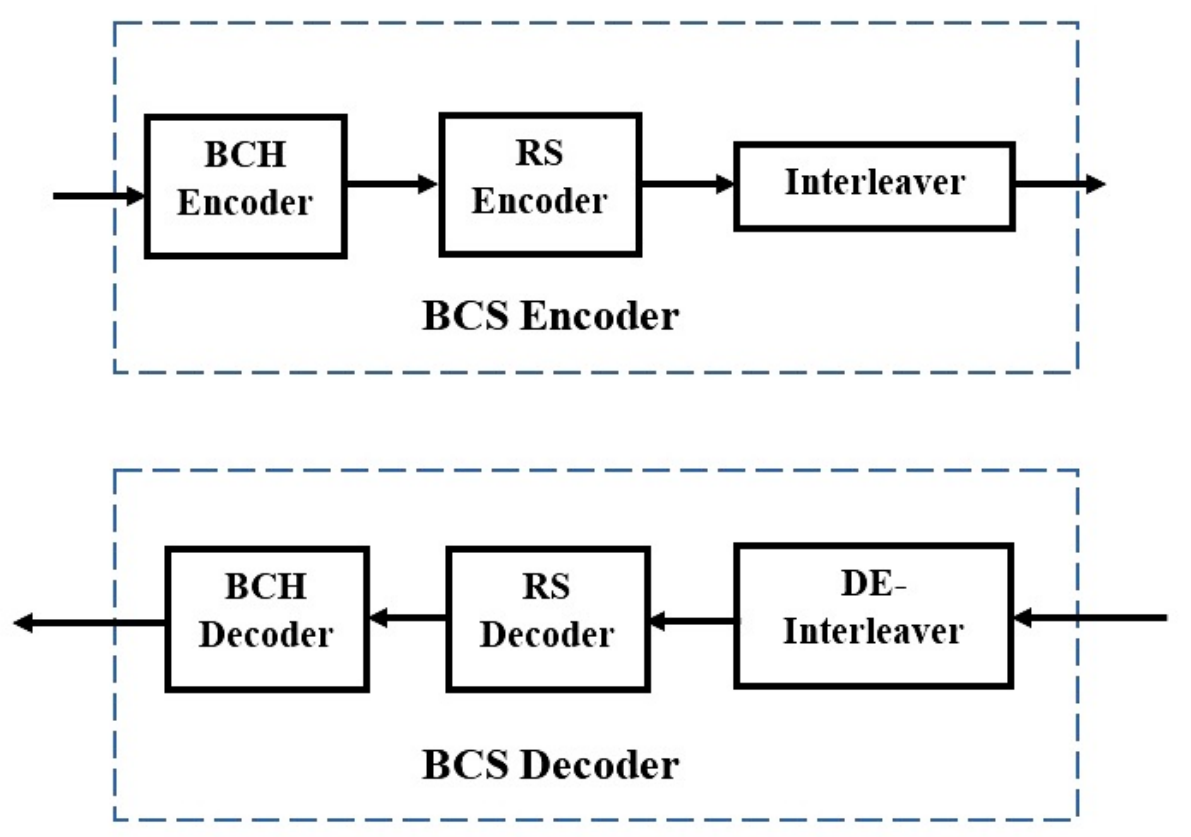

Fig. 1 Novel BCS code

On the other hand, BCS code was suggested in [29] to be used with f-OFDM system and introduced as a promising technique for $5 \mathrm{G}$ systems. They explained that a considerable enhancement in Bit Error Rate (BER) performance and minimized PAPR has been achieved by using BCS code in the f-OFDM system, while 
using finite impulse response (FIR) filter is significantly reducing OOBE. The same idea of BCS code [28] has been used in this paper for concatenated RS/LDPC code. The proposed concatenated RS/LDPC code is created using an inner LDPC code with an outer RS code then appended by interleaver. Thereafter, the proposed concatenated RS/LDPC code will be used with the f-OFDM system and introduced as a candidate for $5 \mathrm{G}$ wireless communication system.

\section{System Diagram}

The proposed concatenated RS/LDPC codes in the f-OFDM system block diagram have been depicted in Fig 2. The simulation is performed by MATLAB software over a 2X2 MIMO system in the presence of a multipath fading channel for BPSK and Quadrature Phase Shift Keying (QPSK) modulation schemes. In this paper, owing to the ease and simplicity of online generation filters, the windowed Sinc scheme is chosen to be used in f-OFDM system architecture among the existing FIR filter schemes. Kaiser, Hanning and Rooted Raised Cosine (RRC) are the different options of window functions. The RRC window has been used in this paper to achieve good trade-off between time and frequency localization [30].

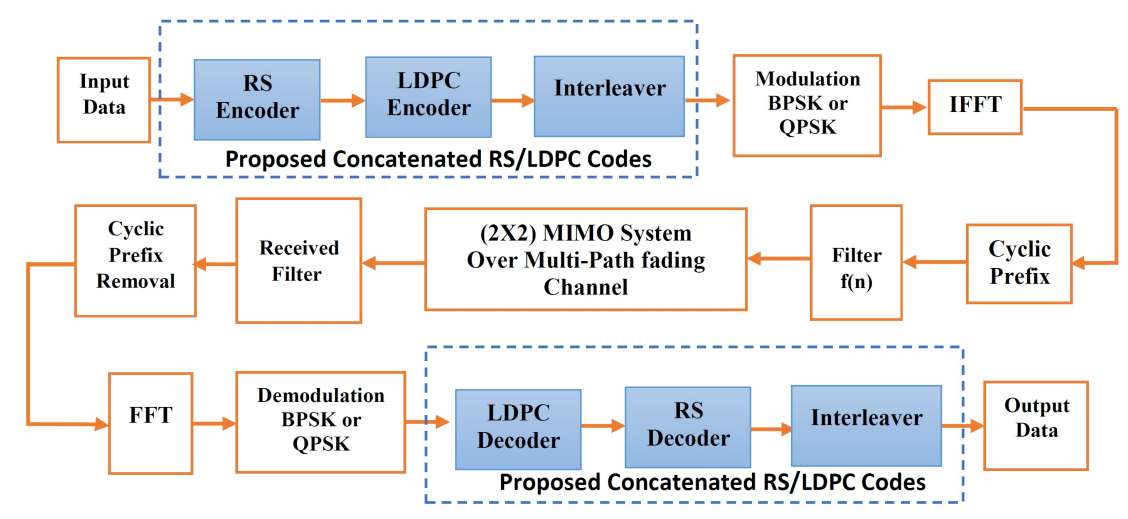

Fig. 2 Proposed System Diagram

As shown in Fig. 2, the discrete binary stream is the input data that will be encoded using the proposed RS/LDPC codes. After that, the encoded data will be modulated using either BPSK or QPSK modulation schemes. The OFDM signal will be created after using both Inverse Fast Fourier Transform (IFFT) and Cyclic Prefix (CP). By using the FIR filter on the OFDM signal, the f-OFDM signal will be created. By using filter $\mathrm{f}(\mathrm{n})$, it is possible to utilize several types of windows functions to obtain f-OFDM [1]. In general, $\mathrm{S}(\mathrm{n})$ is the classic OFDM symbol can be written as follows [1]:

$$
S(t)=\sum_{i=0}^{N-1} d_{i} \operatorname{rect}\left(t-t_{s}-(T / 2)\right) e^{i 2 \pi f_{i}\left(t-t_{s}\right)} \quad t_{s} \leq t \leq t_{s}+T
$$


Table 1 Simulation Parameters

\begin{tabular}{lll}
\hline & Transmission BW & $20 \mathrm{MHZ}$ \\
\hline & Channel & $(2 \mathrm{X} 2)$ MIMO System \\
& Multipath Fading Channel \\
& No.of IFFT/FFT Points & 2048 \\
& No. Occupied Sub-Carriers & 1200 \\
& CP Length & 144 \\
& Modulation Schemes & BPSK and QPSK \\
& Subcarrier Spacing & $15 \mathrm{kHz}$ \\
\hline & B.W & $19.83 \mathrm{MHz}$ \\
Filter & Sampling Rate & $30.72 \mathrm{MHz}$ \\
Design & Type & Root-Raised-Cosine \\
& & $(\mathrm{RRC}) \mathrm{Windowed}-$ Sinc \\
& Roll-off factor $(\alpha)$ & 0.6 \\
\hline Channel Coding & Congth & 513 \\
\hline
\end{tabular}

where $N, d_{i}, T, f_{i}$ and rect(t) is the subcarrier numbers, complex data symbol, symbol duration, the subcarrier frequency and rectangle function, respectively.

Assume that $\left\{s_{n, k}\right\}_{k=0}^{N-1}$ with $E\left|S_{n, k}\right|^{2}=\sigma_{s}^{2}$ the transmitted complex symbols of nth OFDM block. Hence, the OFDM signal could be written as follows [1]:

$$
S_{n}(t)=\sum_{k=0}^{N-1} S_{n, k} e^{j 2 \pi k \Delta f t} \quad 0 \leq t \leq T_{s}
$$

where $\Delta f, T_{s}$ is the sub-channels space and symbol duration.

By passing the OFDM signal $s(n)$ via the filter $f(n)$, the f-OFDM signal $\widetilde{s(n)}$ could be created. Hence, it could be expressed using the convolution process as follows [1]:

$$
\widetilde{s(n)}=S(n) * f(n)
$$

The time domain of the filter is written as follows [30]:

$$
f(n)=S_{i d}(n) * w(n)
$$

where $w(n)$ and $f_{i d}(n)$ is the time domain of the window function and the ideal low pass filter, respectively [30]:

$$
f_{i d}(n)=1 / 2 \int_{-w_{c}}^{w_{c}} e^{j w n} d w=\sin \left(w_{c} n\right) / w_{c} n
$$

where $w_{c}$ is the low pass filter's cutoff frequency While, the time domain of the $\mathrm{RRC}$ window is given by [30] :

$$
W_{R R C}(n)=\left[0.5\left(1-\cos \left(2 \pi n / N_{f}-1\right)\right)\right]^{\alpha}
$$

where $\alpha$ is the factor controlling the window shape ( $\alpha=0.6$ for RRC) and $N_{f}$ represents the filter's length. 


\section{Simulation Results}

In the beginning, BER performance will be evaluated for the proposed concatenated RS/LDPC codes in the f-OFDM system then compared to both single and conventional concatenated RS/LDPC codes for both modulation schemes. The impact of using interleaver in single and concatenated codes will be discussed. Furthermore, using the interleaver between outer and inner codes in conventional RS/LDPC codes against using after both codes in the proposed concatenated $\mathrm{RS} / \mathrm{LDPC}$ codes will also be compared in this section. BER performance of LDPC codes in the f-OFDM system for BPSK is depicted in Fig. 3. It showed that both curves of BER performance for LDPC code with and without interleaver at low values of Signal to Noise Ratio (SNR) were smoothly improving and the errors decreasing. On contrast, they give a constant number of errors at higher SNR values due to the error floor phenomenon. Thus, using interleaver with LDPC code does not help in solving the problem of error floor phenomenon for BPSK modulation schemes.

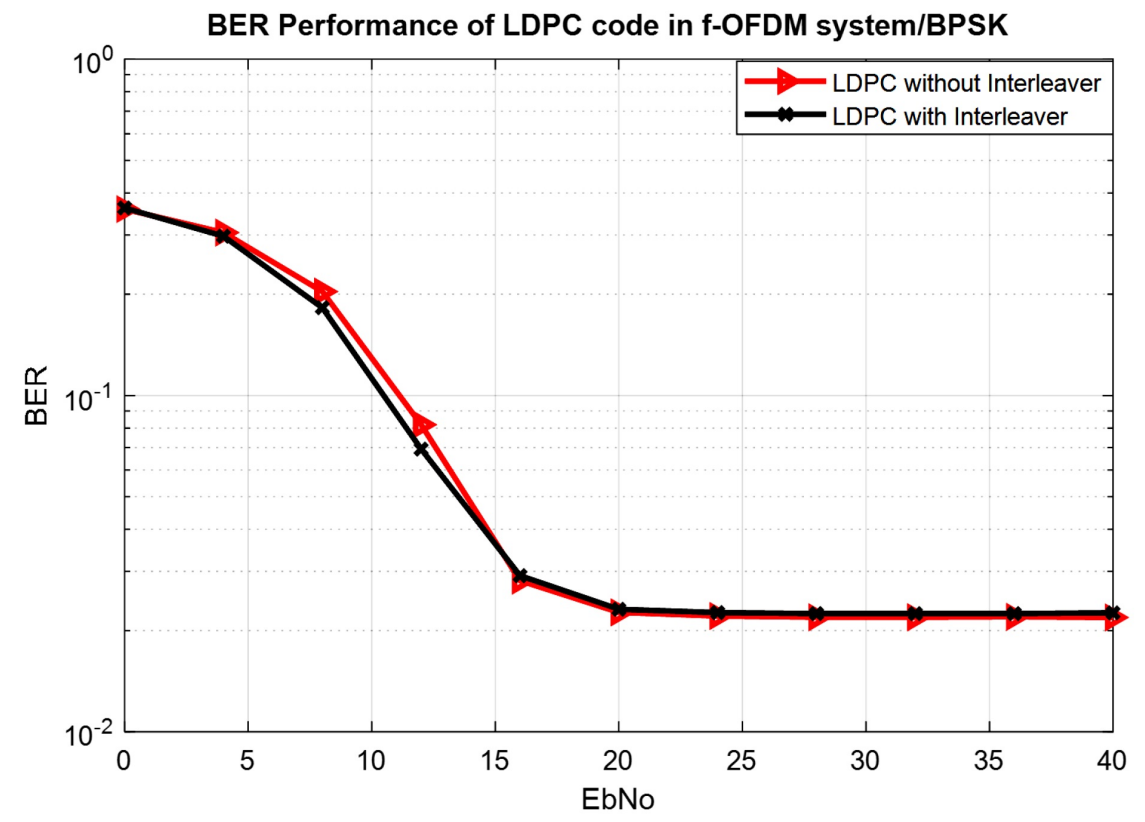

Fig. 3 The Performance of LDPC code in f-OFDM system/BPSK

On the other hand, Fig. 4 shows the BER performance of LDPC codes in the f-OFDM system for QPSK modulation schemes. It shows that, for QPSK, using interleaver could not help in solving the LDPC code's problem. Both curves could not decrease the errors after around $20 \mathrm{~dB}$ and $24 \mathrm{~dB}$ without and with interleaver, respectively. Thus, the error floor problem of LDPC code still exist and could not be solved by using interleaver for both modulation schemes. 


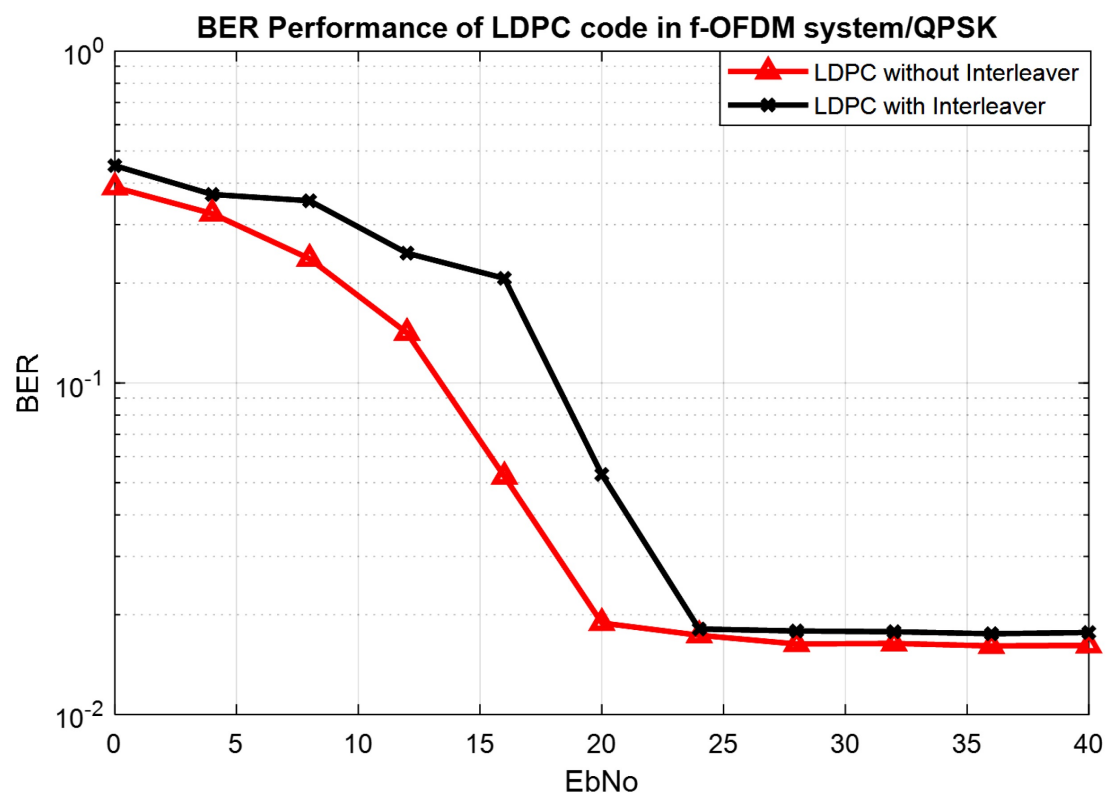

Fig. 4 The Performance of LDPC code in f-OFDM system/QPSK

The performance of the RS code with the f-OFDM system for BPSK is showed in Fig. 5. The outcomes showed that the BER performance of the proposed system using RS code with interleaver outperformed the performance without interleaver. Using interleaver with RS code is significantly improved the BER performance of the f-proposed system and eliminated the error floor compared to use RS code without interleaver for BPSK. Thus, around $15 d B$ coding gain has been achieved at $2 \times 10^{-2}$ by using interleaver compared to RS codes without interleaver for the BPSK. Also, for the QPSK modulation scheme, using interleaver with RS code in f-OFDM system significantly improves BER performance and eliminates the error floor compared to use only RS code in the f-OFDM system without interleaver, as depicted in Fig 6 . Around $10 d B$ coding gain has been achieved at $2 \times 10^{-2}$ using interleaver compared to RS codes without interleaver for QPSK modulation scheme

Therefore, using interleaver with RS codes has significantly improved the BER system performance and eliminated the error floor phenomenon, unlike the LDPC codes, which does not improve the BER performance or eliminate the error floor phenomenon using the interleaver. Thus, the LDPC code needs to be used with other error correction codes in concatenated form to solve its error floor phenomenon. The performance of conventional concatenated RS/LDPC code that uses outer RS code with inner LDPC code then separated by interleaver in the f-OFDM system is depicted in Fig. 7 for the BPSK. It is clear that using conventional concatenated RS/LDPC code in the f-OFDM system outperforms both single codes. Using concatenated code achieved lower BER than single RS and LDPC codes for BPSK. Meanwhile, BER performance of conventional concatenated RS 


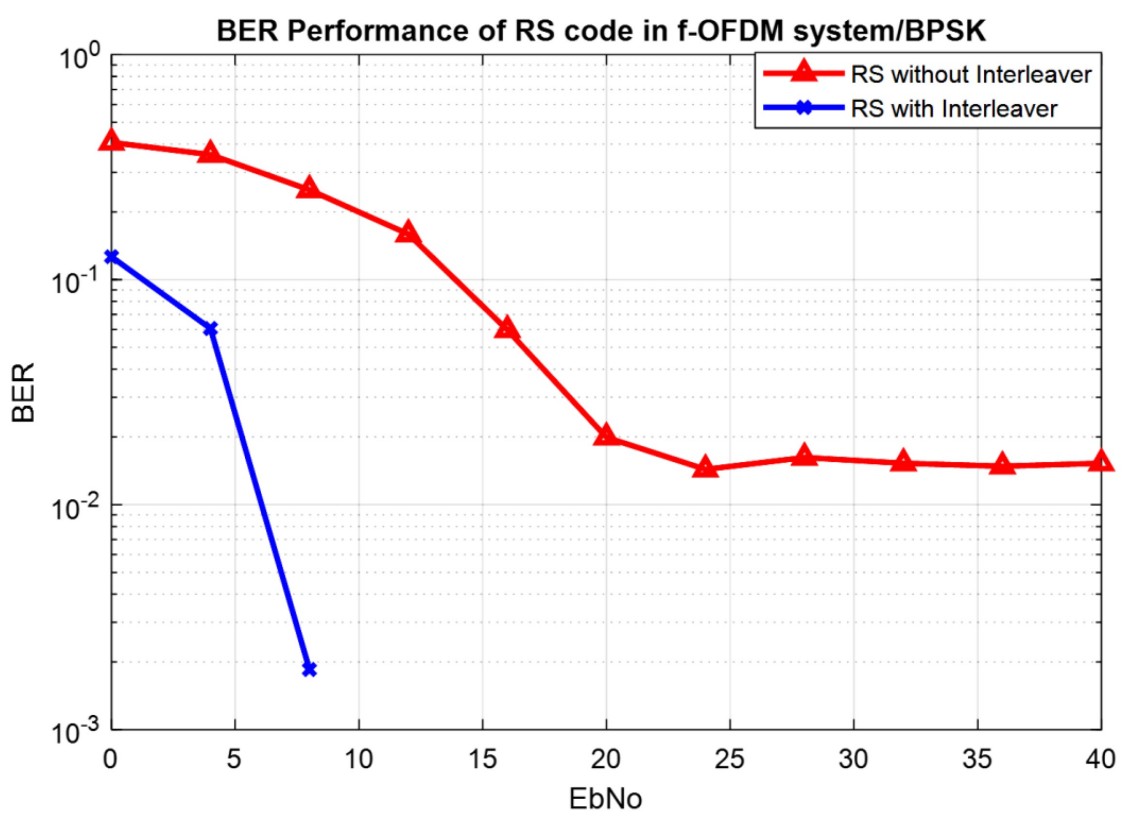

Fig. 5 The Performance of RS code in f-OFDM system/BPSK

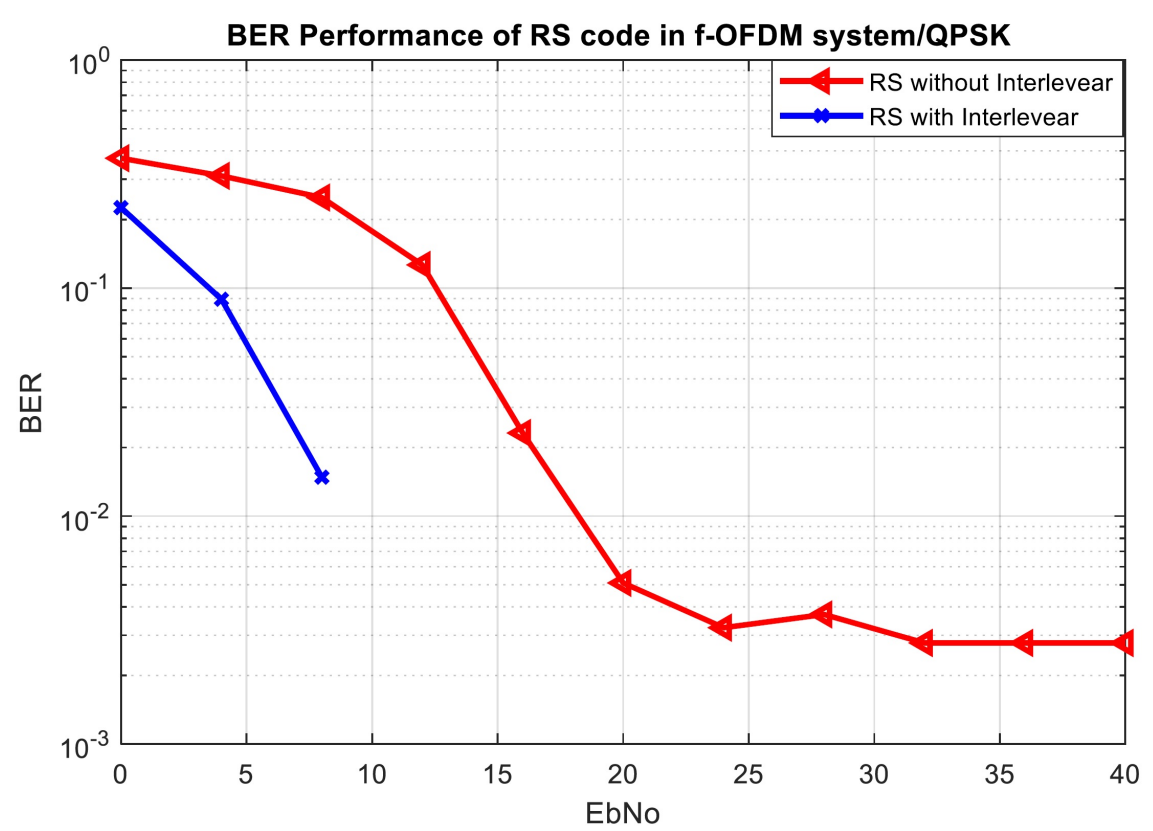

Fig. 6 The Performance of RS code in f-OFDM system/QPSK

/LDPC code in f-OFDM system for QPSK modulation scheme is depicted in Fig. 
8. Also, for QPSK, the familiar concatenated RS/LDPC code outperforms single RS and LDPC codes. Thus, using LDPC code with RS code in concatenated code scheme achieved significant improvement in the f-OFDM system in terms of BER performance better than single RS and LDPC code and contributes in solving the error floor phenomenon of LDPC code in both BPSK and QPSK modulation schemes. Here, around 12 and $17 d B$ coding gain at $3 \times 10^{-2}$ have been achieved by using conventional concatenated RS/LDPC code against a single LDPC code in BPSK and QPSK, respectively.

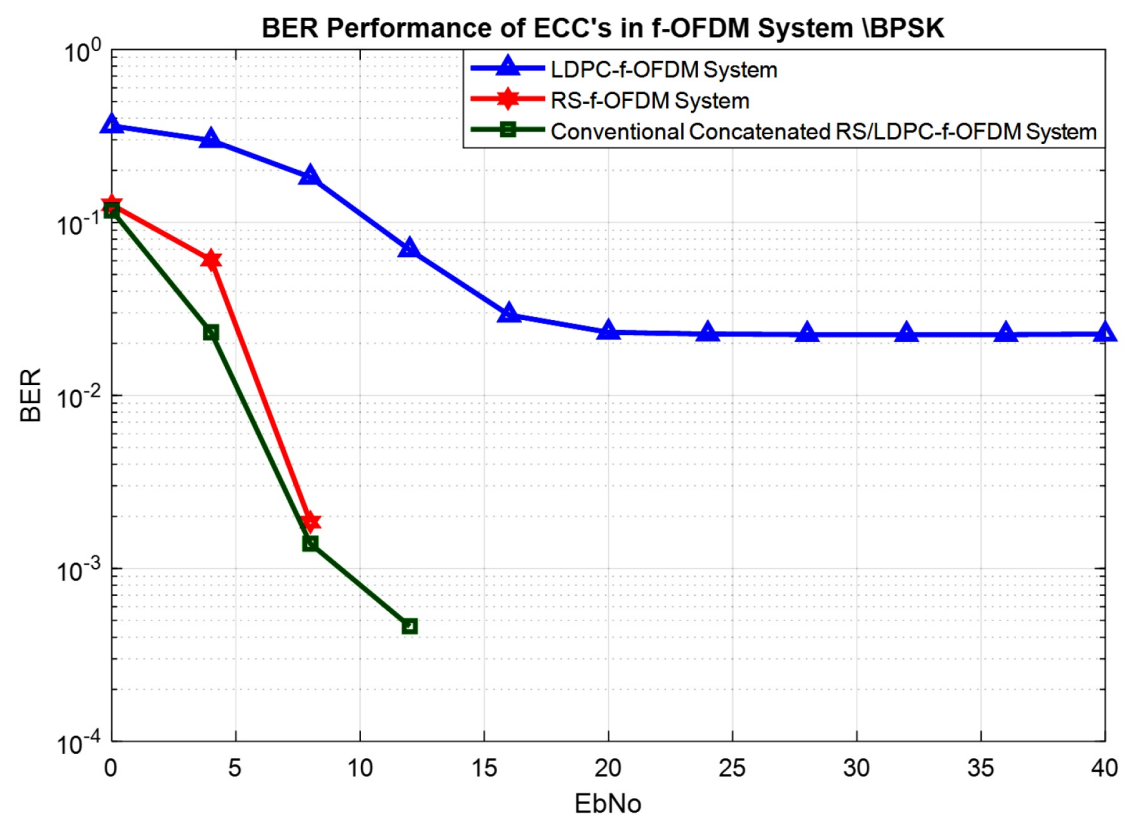

Fig. 7 The Performance of ECC's in f-OFDM system/BPSK

The modified concatenated RS/LDPC codes which use outer RS code with inner LDPC code and appended by interleaver has been suggested for the fOFDM system in this paper and compared to single and conventional concatenated RS/LDPC code for both modulation scheme as depicted in Fig. 9 and 10. In both modulation schemes, although the conventional concatenated RS/LDPC codes that used the interleaver between both codes outperform single RS and LDPC codes, achieving lower BER and terminating the error floor phenomenon of LDPC codes shown in Fig. 7 and 8. Nevertheless, the proposed modified concatenated RS/LDPC codes that used the interleaver after both codes outperformed the single and conventional concatenated RS/LDPC codes for both modulation schemes, as shown in Fig. 9 and 10.

Due to the use of the interleaver after both outer and inner codes, the modified concatenated RS/LDPC codes outperformed conventional concatenated RS/LDPC codes. By using this method, the interleaver helps both outer and inner decoders 


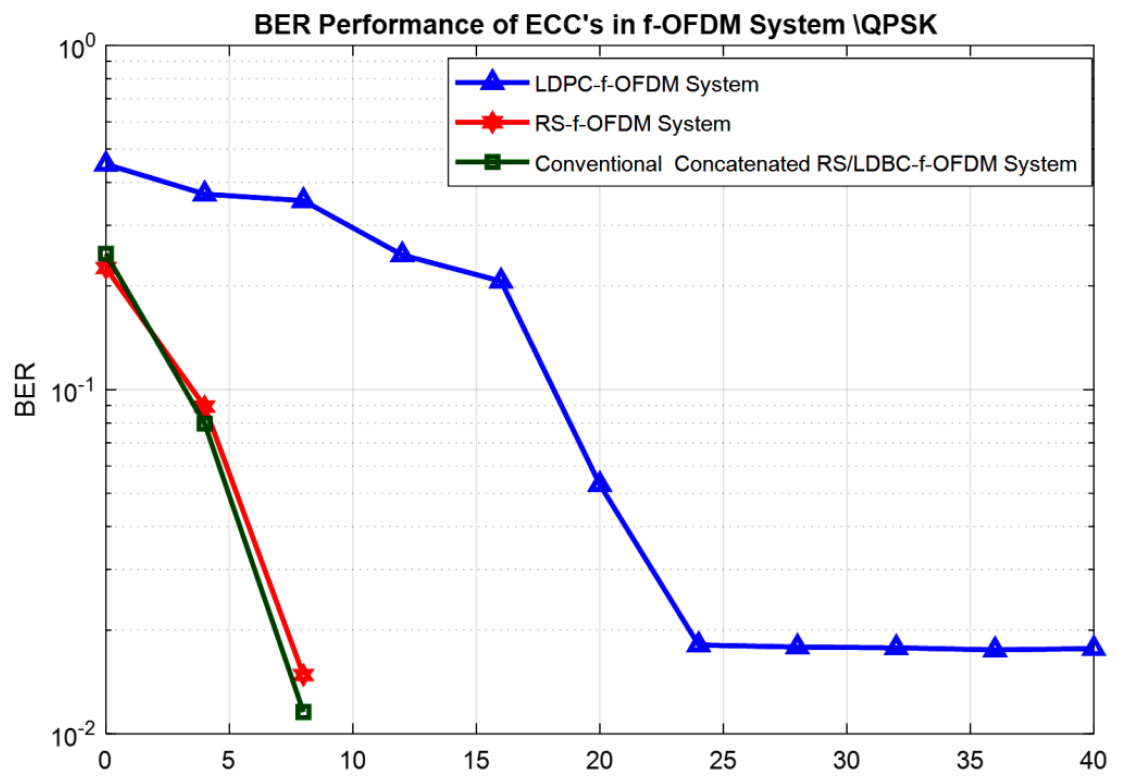

Fig. 8 The Performance of ECC's in f-OFDM system/QPSK

in correcting the errors, unlike the conventional method that uses interleaver in between, which helps only the outer decoder in correcting the errors.

The results proved that using the modified concatenated RS/LDPC codes in f-OFDM system achieved significant improvements in terms of BER performance, outperforming single and conventional concatenated RS/LDPC codes in both modulation schemes. Meanwhile, the Power Spectrum Density (PSD) of both f-OFDM and familiar OFDM systems using proposed concatenated RS/LDPC codes are compared in Fig. 11 and 12 for BPSK and QPSK, respectively. The outcomes showed that the OOBE of the f-OFDM system is lower than the familiar OFDM system in both modulation schemes. It achieved around 80dB OOBE values lower than the familiar OFDM system in both modulation schemes. Thus, owing to use the FIR filter in the f-OFDM system contributed in decreasing OOBE values to be significantly lower than the familiar OFDM system as shown in Figs. 11 and 12. Therefore, the proposed system not only enhances the performance of the f-OFDM system but also maintains the OOBE levels at low values.

The last evaluation of the system performance is the PAPR values and compared to the conventional OFDM system. The comparison of PAPR values for the proposed system versus the OFDM system are depicted in Table 2.

Table 2 PAPR values of the proposed system versus Familiar OFDM system

\begin{tabular}{lll}
\hline Technique & Familiar OFDM system & Proposed system \\
\hline BPSK & $10.1926 \mathrm{~dB}$ & $10.5502 \mathrm{~dB}$ \\
\hline QPSK & $10.1961 \mathrm{~dB}$ & $10.055 \mathrm{~dB}$ \\
\hline
\end{tabular}




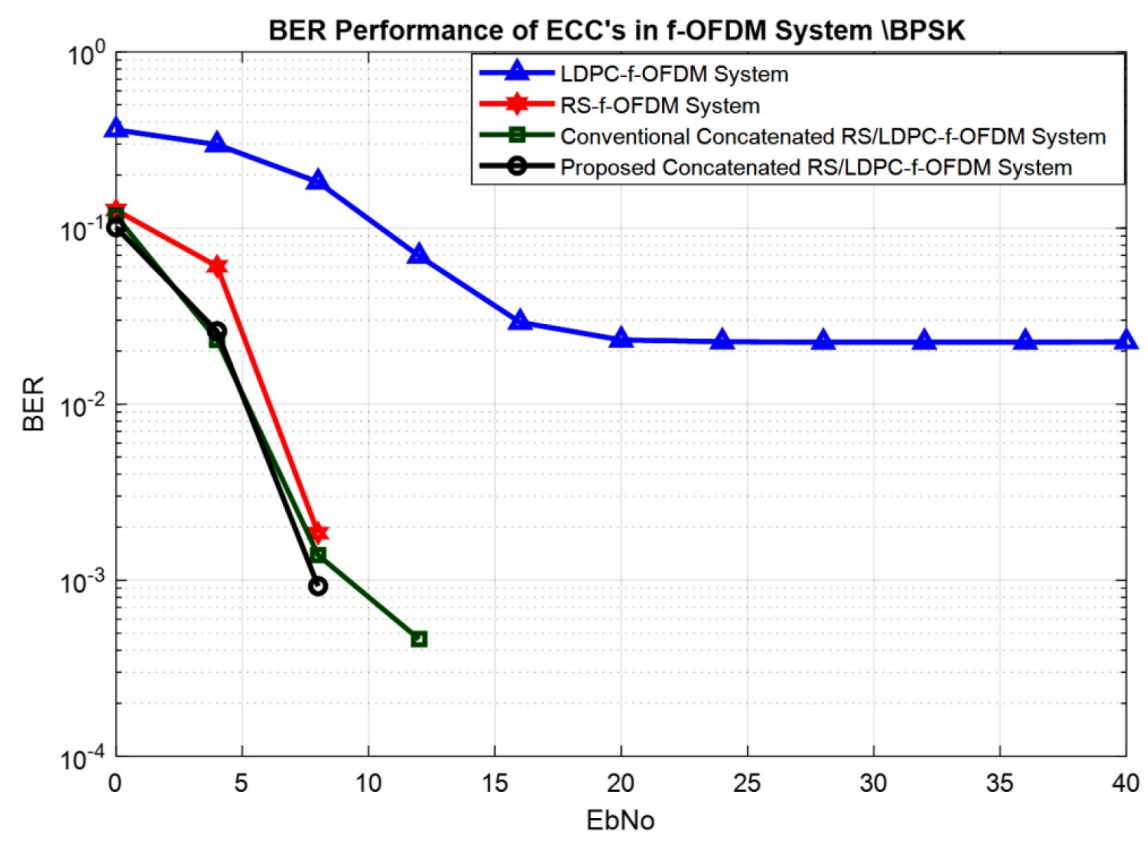

Fig. 9 The Performance of proposed concatenated RS/LDPC code in f-OFDM system/BPSK

Based on the results of both systems, it can be concluded that the suggested system could save the PAPR values near or lower the conventional OFDM system for both modulation schemes. Both systems achieved somewhat close values. Thus, the proposed system can be introduced, which is designed using modified concatenated RS/LDPC code in the $\mathrm{f}-\mathrm{OFDM}$ system as an alternative waveform to conventional OFDM system. This is due to solving the problem of high OOBE level using FIR filter and achieving PAPR values near of conventional OFDM system. Furthermore, the use of the modified concatenated RS/LDPC codes in the f-OFDM system contributed to enhancing the BER performance to be better than both single and conventional concatenated RS/LDPC codes. This, led to introducing the proposed system as a contender waveform for $5 \mathrm{G}$ wireless communication systems due to these features.

\section{Conclusion}

The modified concatenated RS/LDPC codes are suggested in this paper for the f-OFDM system via a multipath fading channel using the 2 X2MIMO system. The proposed concatenated code in this paper is created using inner LDPC code with outer RS code then appended by interleaver unlike the conventional concatenated code that separated the outer and inner codes by interleaver. The impact of using the interleaver on single RS and LDPC codes has also been discussed. The results showed that LDPC codes' performance did not improve, and the error floor phenomenon still exists even with using interleaver. Unlike the RS codes, which 


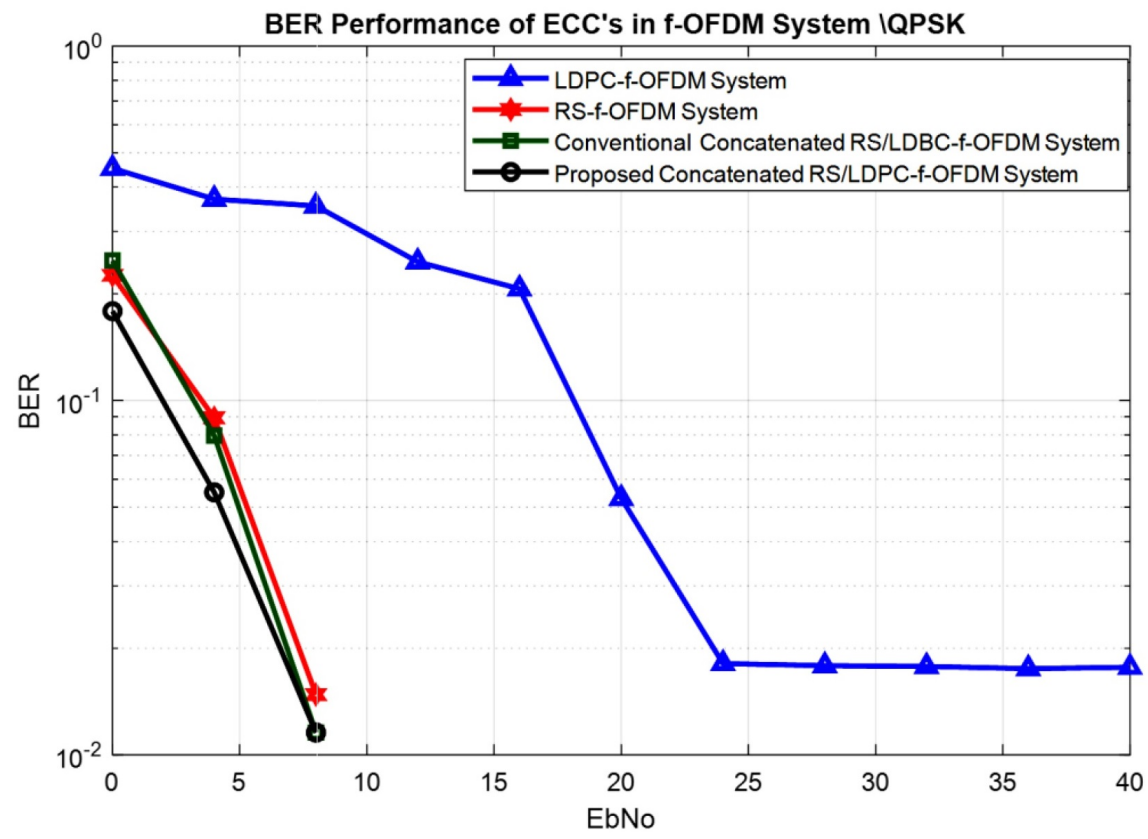

Fig. 10 The Performance of proposed concatenated RS/LDPC code in f-OFDM system/QPSK
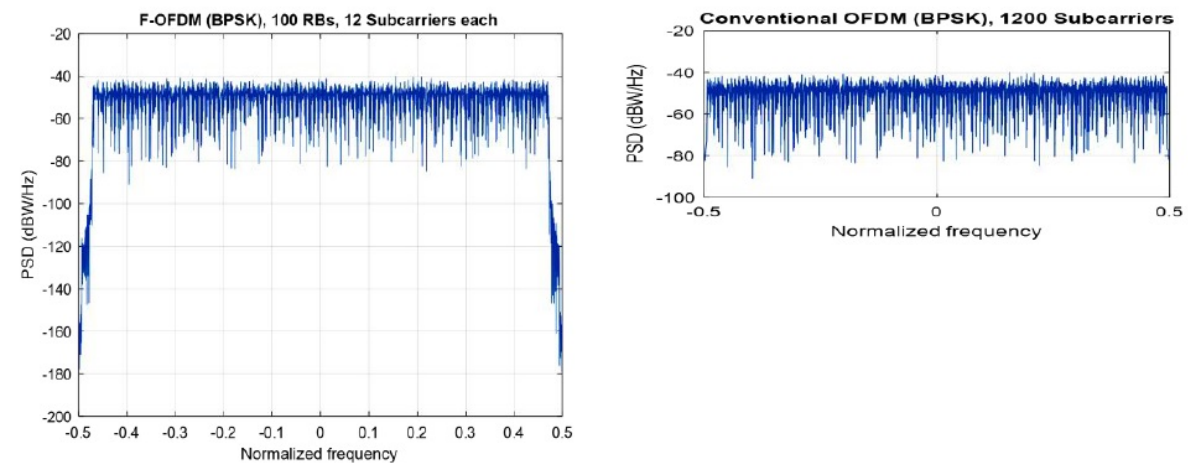

Fig. 11 PSD of OFDM versus proposed system/BPSK

are significantly improved and the phenomenon of the error floor is eliminated by using interleaver. Around 15 and $10 \mathrm{~dB}$ coding gain have been achieved at $2 \times 10^{-2}$ by using RS with interleaver compared to the RS codes without interleaver for BPSK and QPSK, respectively. On the other hand, the conventional concatenated RS/LDPC codes in the f-OFDM system outperformed single codes in terms of BER performance. Nevertheless, the proposed modified concatenated RS/LDPC codes outperformed single and conventional concatenated RS/LDPC 

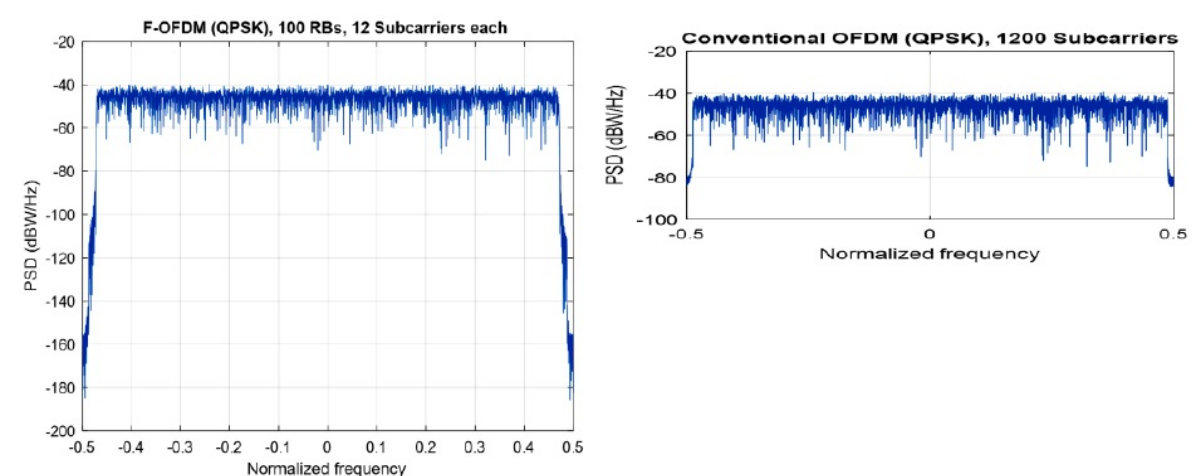

Fig. 12 PSD of OFDM versus proposed system/QPSK

codes in both modulation schemes. Meanwhile, using LDPC codes with RS codes in concatenated form contributed to improving the BER performance and eliminating the phenomenon of error floor of LDPC codes. Around 12 and $17 \mathrm{~dB}$ coding gain at $3 \times 10^{-2}$ have been achieved using concatenated RS/LDPC code against a single LDPC code in BPSK and QPSK, respectively. In contrast, due to the use of the FIR filter in the suggested system, the f-OFDM system using modified concatenated RS/LDPC codes achieved around $80 \mathrm{~dB}$ lower values of OOBE than the conventional OFDM system. It also recorded near or lower values PAPR of the conventional OFDM system. In conclusion, the suggested system is a promising contender for $5 \mathrm{G}$ wireless communication systems.

Availability of Data and Material

Data sharing not applicable to this article as all results have been obtained using MATLAB simulation during the current study.

\section{Competing interests}

The author declare that they have no competing interests.

Fundin

Not applicable.

Authors' contributions

All authors read and approved the final manuscript.

Acknowledgements

Not applicable.

Authors' information

Ghasan Ali Hussain has a Ph.D. from Universiti Tun Hussein Onn Malaysia in 2021. He received the Master degree in communication Engineering from University Putra Malaysia in 2013.Hussain received Bachelor degree in Communication Engineering from Alfatah University- Tripoli/Libya in 2003. Since 2006, he has been working at Electrical Engineering, University of Kufa, Iraq. His research in- 
terests include wireless communication systems, Mobile systems, LTE and Error Correction Techniques. He has a number of publications in different journals in his field.

\section{References}

1. X. Cheng, Y. He, B. Ge, C. He, in 2016 IEEE 83rd Vehicular Technology Conference (VTC Spring) (2016), pp. 1-5. DOI 10.1109/VTCSpring.2016.7504065

2. S. Cheng, in Proc. 3rd Intl. Conf. Mechatronics Engineering and Information Technology (2019)

3. G.A. Hussain, L. Audah, Bch codes for $5 \mathrm{~g}$ wireless communication systems over multipath fading channel, indonesian journal of electrical engineering and computer science 17(1), $310(2020)$

4. G.A. Hussain, L. Audah, Rs codes with filtered-ofdm: a waveform contender for $5 \mathrm{~g}$ mobile communication systems, wireless personal communications 115(1), 575 (2020)

5. G.A. Hussain, L. Audah, Ufmc system performance improvement using rs codes for $5 \mathrm{~g}$ communication system, Telkomnika 18(4), 1843 (2020)

6. G.A. Hussain, L. Audah, Bch codes in ufmc: A new contender candidate for $5 \mathrm{~g}$ communication systems, Bulletin of Electrical Engineering and Informatics 10(2), 904 (2021)

7. M. Dhuheir, S. Ztrk, Polar codes applications for $5 \mathrm{~g}$ systems, Journal of Institue Of Science and Technology 34(3) (2018)

8. P. Chen, M. Xu, B. Bai, J. Wang, in 2017 IEEE 85th Vehicular Technology Conference (VTC Spring) (2017), pp. 1-5. DOI 10.1109/VTCSpring.2017.8108615

9. A. Sharma, M. Salim, in 2017 International Conference on Computer, Communications and Electronics (Comptelix) (2017), pp. 676-682. DOI 10.1109/COMPTELIX.2017.8004055

10. M. Sybis, K. Wesolowski, K. Jayasinghe, V. Venkatasubramanian, V. Vukadinovic, in 2016 IEEE 84th Vehicular Technology Conference (VTC-Fall) (2016), pp. 1-5. DOI 10.1109/VTCFall.2016.7880930

11. R.G. Maunder, The $5 \mathrm{~g}$ channel code contenders, ACCELERCOMM white paper pp. 1-13 (2016)

12. B. Zhang, H. Shen, B. Yin, L. Lu, D. Chen, T. Wang, L. Gu, X. Wang, X. Hou, H. Jiang, A. Benjebbour, Y. Kishiyama, in 2016 IEEE Globecom Workshops (GC Wkshps) (2016), pp. 1-6. DOI 10.1109/GLOCOMW.2016.7848800

13. J.H. Bae, A. Abotabl, H.P. Lin, K.B. Song, J. Lee, An overview of channel coding for $5 \mathrm{~g}$ nr cellular communications, APSIPA Transactions on Signal and Information Processing $8(2019)$

14. D. Hui, S. Sandberg, Y. Blankenship, M. Andersson, L. Grosjean, Channel coding in 5g new radio: A tutorial overview and performance comparison with $4 \mathrm{~g}$ lte, IEEE Vehicular Technology Magazine 13(4), 60 (2018). DOI 10.1109/MVT.2018.2867640

15. Z.R.M. Hajiyat, A. Sali, M. Mokhtar, F. Hashim, Channel coding scheme for $5 \mathrm{~g}$ mobile communication system for short length message transmission, Wireless Personal Communications 106(2), 377 (2019)

16. K. Arora, J. Singh, Y.S. Randhawa, A survey on channel coding techniques for $5 \mathrm{~g}$ wireless networks, Telecommunication Systems 73(4), 637 (2020)

17. H. Mao, Q. Li, Q. Han, H. Guo, High-throughput and low-cost ldpc reconciliation for quantum key distribution, Quantum Information Processing 18(7), 1 (2019)

18. Y. Han, W.E. Ryan, Low-floor decoders for ldpc codes, IEEE Transactions on Communications 57(6), 1663 (2009). DOI 10.1109/TCOMM.2009.06.070325

19. S.L. Shieh, Concatenated bch and ldpc coding scheme with iterative decoding algorithm for flash memory, IEEE Communications Letters 19(3), 327 (2015). DOI 10.1109/LCOMM.2015.2391260

20. Y. Zhang, W.E. Ryan, Toward low ldpc-code floors: a case study, IEEE Transactions on Communications 57(6), 1566 (2009). DOI 10.1109/TCOMM.2009.06.0700712

21. J. Oh, J. Ha, H. Park, J. Moon, Rs-ldpc concatenated coding for the modern tape storage channel, IEEE Transactions on Communications 64(1), 59 (2016). DOI 10.1109/TCOMM.2015.2504362 
22. P.J. Nadkarni, S.S. Garani, Entanglement-assisted reed-solomon codes over qudits: theory and architecture, Quantum Information Processing 20(4), 1 (2021)

23. T. Mizuochi, Y. Konishi, Y. Miyata, T. Inoue, K. Onohara, S. Kametani, T. Sugihara, K. Kubo, H. Yoshida, T. Kobayashi, et al., Experimental demonstration of concatenated ldpc and rs codes by fpgas emulation, IEEE Photonics Technology Letters 21(18), 1302 (2009)

24. S. Jeon, X. Hu, B.V. Kumar, in IEEE GLOBECOM 2008-2008 IEEE Global Telecommunications Conference (IEEE, 2008), pp. 1-5

25. J. Qiu, L. Chen, S. Liu, A novel concatenated coding scheme: Rs-sc-ldpc codes, IEEE Communications Letters 24(10), 2092 (2020). DOI 10.1109/LCOMM.2020.3004917

26. J. Qiu, S. Liu, L. Chen, in 2019 11th International Conference on Wireless Communications and Signal Processing (WCSP) (2019), pp. 1-6. DOI 10.1109/WCSP.2019.8928034

27. A. Suls, J. Neckebroek, Y. Lefevre, M. Guenach, M. Moeneclaey, in 2016 Symposium on Communications and Vehicular Technologies (SCVT) (2016), pp. 1-6. DOI 10.1109/SCVT.2016.7797667

28. G.A. Hussain, L. Audah, A novel bcs code in a downlink lte system over an lte-mimo channel, Telecommunication Systems 74(4), 467 (2020)

29. G.A. Hussain, L. Audah, A bcs code in the f-ofdm system: a promising candidate for $5 \mathrm{~g}$ wireless communication systems, Journal of Ambient Intelligence and Humanized Computing pp. 1-10 (2021)

30. D. Wu, X. Zhang, J. Qiu, L. Gu, Y. Saito, A. Benjebbour, Y. Kishiyama, in 2016 IEEE Globecom Workshops (GC Wkshps) (2016), pp. 1-6. DOI 10.1109/GLOCOMW.2016.7848810 